\title{
Gröbner-Shirshov bases for free partially commutative Lie algebras *
}

\author{
Yuqun Chen and Qiuhui Mo \\ School of Mathematical Sciences, South China Normal University \\ Guangzhou 510631, P.R. China \\ yqchen@scnu.edu.cn \\ scnuhuashimomo@126.com
}

\begin{abstract}
In this paper, by using Composition-Diamond lemma for Lie algebras, we give a Gröbner-Shirshov basis for free partially commutative Lie algebra over a commutative ring with unit. As an application, we obtain a normal form for such a Lie algebra.
\end{abstract}

Key words: Gröbner-Shirshov basis; Lie algebra; partially commutative algebra, normal form.

AMS 2010 Subject Classification: 17B01, 16S15, 13P10

\section{Introduction}

The free partially commutative monoid was introduced by P. Cartier and D. Foata in 1969 [3] for the study of combinatorial problems in connection with word rearrangements. Since that time, this monoid has been the subject of many studies. They were principally motivated by the fact that the free partially commutative monoid is a model for concurrent computing. On the other hand, they can be seen as a natural generalization of free monoids. Indeed, several classical results of the free monoid theory can be extended to the partially commutative framework. For instance, the free partially commutative group, the free partially commutative associative algebra and the free partially commutative Lie algebra. This research direction was followed by a lot of people (see [5, 6, 7, 8, 9, 15, 18] or [13]).

In 1992, G. Duchamp 9] proved that the free partially commutative Lie algebra is a free $K$-module, where $K$ is a commutative ring, and gave an algorithm to find a basis for any free partially commutative Lie algebras. In 1993, G. Duchamp and D. Krob [10] showed how to obtain decomposition results for free partially commutative Lie algebra into free Lie algebras and obtained a normal form for such algebra. The algorithms given by these two papers are based on a decomposition of the generating set of the free partially commutative Lie algebra by two subsets one of which is independent, and a linear basis is implicitly given.

In 2001, by using Composition-Diamond lemma for associative algebras, L.A. Bokut and L.S. Shiao [2] gave Gröbner-Shirshov bases for free partially commutative associative

\footnotetext{
${ }^{*}$ Supported by the NNSF of China (Nos. 10771077; 10911120389).
} 
algebras and free partially commutative monoids. In 2004, E.S. Esyp, I.V. Kazatchkov and V.N. Remeslennikov [11] gave a Gröbner-Shirshov basis for free partially commutative group. In this paper, by using Composition-Diamond lemma for Lie algebras, we give a Gröbner-Shirshov basis for free partially commutative Lie algebra over a commutative ring with unit. As an application, we obtain a linear basis explicitly for such a Lie algebra. E.N. Poroshenko [14] also gives the same Gröbner-Shirshov basis for free partially commutative Lie algebra independently. Our proof is different from [14].

Let $X$ be a set, $K$ a commutative ring with unit and $\operatorname{Lie}(X)$ the free Lie algebra over $K$ generated by $X$. Let $\vartheta \subseteq(X \times X) \backslash\{(x, x) \mid x \in X\}$. Then $\operatorname{Lie}(X \mid \vartheta)=\operatorname{Lie}(X) / \operatorname{Id}(\vartheta)$ is free partially commutative Lie algebra, where $\operatorname{Id}(\vartheta)$ is the ideal of $\operatorname{Lie}(X)$ generated by the set $\{(a b) \mid(a, b) \in \vartheta\}$ and $(a b)$ is the Lie multiplication in $\operatorname{Lie}(X)$.

\section{Preliminaries}

We start with the associative Lyndon-Shirshov words.

Let $X=\left\{x_{i} \mid i \in I\right\}$ be a well-ordered set with $x_{i}>x_{p}$ if $i>p$ for any $i, p \in I$ and $X^{*}$ the free monoid generated by $X$. We order $X^{*}$ by the lexicographical ordering.

Definition 2.1 ([1, 4, 12, 16, 17, 19]) An associative word $u(u \neq 1)$ in $X$ is called an ALSW (associative Lyndon-Shirshov word) if

$$
\left(\forall v, w \in X^{*}, v, w \neq 1\right) u=v w \Rightarrow v w>w v .
$$

A non-associative word $(u)$ in $X$ is called a NLSW (non-associative Lyndon-Shirshov word) if

(i) $u$ is an $A L S W$,

(ii) if $(u)=((v)(w))$, then both $(v)$ and $(w)$ are $N L S W ' s$,

(iii) in (ii) if $(v)=\left(\left(v_{1}\right)\left(v_{2}\right)\right)$, then $v_{2} \leq w$ in $X^{*}$.

Lemma 2.2 (4, 16, 17]) (i) For any $u \in X^{*}$, there exists a unique decomposition $u=$ $u_{1} u_{2} \cdots u_{k}$, where $u_{i}$ is an $A L S W, 1 \leq i \leq k$, and $u_{1} \leq u_{2} \leq \cdots \leq u_{k}$.

(ii) Let $u$ be an $A L S W$ and $|u| \geq 2$. If $u=v w$, where $w$ is the longest $A L S W$ proper end of $u$, then $v$ is an $A L S W$.

Following [4, 16], for an $A L S W u$, there is a unique bracketing, denoted by [u], such that $[u]$ is $N L S W$ :

$$
\left[x_{i}\right]=x_{i},[u]=[[v][w]],
$$

where $u=v w$ and $w$ is the longest $A L S W$ proper end of $u$.

Now, we consider ( ) as Lie bracket in the free associative algebra $k\langle X\rangle$, i.e., for any $a, b \in k\langle X\rangle,(a b)=a b-b a$, where $k$ is a field. We may view $\operatorname{Lie}(X)$ as the subLie-algebra of $k\langle X\rangle$ generated by $X$.

For any polynomial $f \in k\langle X\rangle, f$ has the leading word $\bar{f}$. We call $f$ monic if the coefficient of $\bar{f}$ is 1 . By $\operatorname{deg}(f)$ we denote the degree of $\bar{f}$. 
Lemma 2.3 ([1, 4, 12, 16, 17, 19]) $N L S W$ 's forms a linear basis of Lie $(X)$.

Lemma 2.4 (44, 16, 17]) Let [u] be a NLSW. If we consider [u] as a polynomial in $k\langle X\rangle$, then $\overline{[u]}=u$.

Lemma 2.5 ([16, 17]) Let $u, v$ be $A L S W^{\prime} s, u=a v b, a, b \in X^{*}$. Then $[u]=[a[v c] d]$, where $b=c d, c, d \in X^{*}$. Denote by

$$
[u]_{v}=\left.[u]\right|_{\left.[v c] \mapsto\left[[v]\left[c_{1}\right]\right] \cdots\left[c_{k}\right]\right]},
$$

where $c=c_{1} \cdots c_{k}, c_{j}$ is an $A L S W$ and $c_{1} \leq c_{2} \leq \cdots \leq c_{k}$. Then $\overline{[u]_{v}}=u$.

Let $S \subset \operatorname{Lie}(X)$ with each $s \in S$ monic, $a, b \in X^{*}$ and $s \in S$. If $a \bar{s} b$ is an ALSW, then we define $[a s b]_{\bar{s}}=\left.[a \bar{s} b]_{\bar{s}}\right|_{[\bar{s}]_{\mapsto s}}$, where $[a \bar{s} b]_{\bar{s}}$ is defined by Lemma 2.5 (see [1]).

From now on, we use the deg-lex ordering $<$ on $X^{*}$ : to compare two words by degree first and then lexicographically.

Let $f$ and $g$ be two monic Lie polynomials in $\operatorname{Lie}(X) \subset k\langle X\rangle$. Then, there are two kinds of Lie compositions:

(i) If $w=\bar{f}=a \bar{g} b$ for some $a, b \in X^{*}$, then the polynomial $(f, g)_{w}=f-[a g b]_{\bar{g}}$ is called the composition of inclusion of $f$ and $g$ with respect to $w$.

(ii) If $w$ is a word such that $w=\bar{f} b=a \bar{g}$ for some $a, b \in X^{*}$ with $\operatorname{deg}(\bar{f})+\operatorname{deg}(\bar{g})>\operatorname{deg}(w)$, then the polynomial $(f, g)_{w}=[f b]_{\bar{f}}-[a g]_{\bar{g}}$ is called the composition of intersection of $f$ and $g$ with respect to $w$.

The $w$ in the above is called ambiguity.

Let $S \subset \operatorname{Lie}(X)$ with each $s \in S$ monic.

Suppose that $a, b \in X^{*}$ and $s \in S$. If $\overline{(a s b)}=a \bar{s} b$ and $a \bar{s} b$ is an $A L S W$, then we call $(a s b)$ a normal $s$-word (or normal $S$-word).

Suppose that $w \in X^{*}$ and $h$ is a Lie polynomial. Then $h$ is trivial modulo $(S, w)$, denoted by $h \equiv 0 \bmod (S, w)$, if $h=\sum_{i} \alpha_{i}\left(a_{i} s_{i} b_{i}\right)$, where each $\alpha_{i} \in k, a_{i}, b_{i} \in X^{*}, s_{i} \in S$, and $\left(a_{i} s_{i} b_{i}\right)$ is normal $S$-word such that $a_{i} \overline{s_{i}} b_{i}<w$.

The set $S$ is called a Gröbner-Shirshov basis in $\operatorname{Lie}(X)$ if any composition in $S$ is trivial modulo $S$ and corresponding $w$.

Lemma 2.6 ([16, 17], Composition-Diamond lemma for Lie algebras) Let $S \subset$ Lie $(X)$ be nonempty set of monic Lie polynomials. Let $\operatorname{Id}(S)$ be the ideal of Lie $(X)$ generated by $S$. Then the following statements are equivalent.

(i) $S$ is a Gröbner-Shirshov basis in Lie $(X)$.

(ii) $f \in \operatorname{Id}(S) \Rightarrow \bar{f}=a \bar{s} b$ for some $s \in S$ and $a, b \in X^{*}$.

(iii) $\operatorname{Irr}(S)=\left\{[u] \mid[u]\right.$ is a NLSW, $\left.u \neq a \bar{s} b, s \in S, a, b \in X^{*}\right\}$ is a k-basis for $\operatorname{Lie}(X \mid S)=\operatorname{Lie}(X) / \operatorname{Id}(S)$.

Remark: In this section, if the field $k$ is replaced by a commutative ring $K$ with unit, then all results hold. In particular, the Lemma 2.6 is true for the free Lie algebra over $K$. 


\section{Free partially commutative Lie algebra}

Let $<$ be a well ordering on $X$. Throughout this paper, if $a>b$ and $(a, b) \in \vartheta$ or $(b, a) \in \vartheta$, we denote $a \triangleright b$. Generally, for any set $Y, a \triangleright Y$ means $a \triangleright y$ for any $y \in Y$ and $a>Y$ means $a>y$ for any $y \in Y$. For any $u=x_{i_{1}} \cdots x_{i_{n}} \in X^{*}$ where $x_{i_{j}} \in X$, we denote the set $\left\{x_{i_{j}}, j=1, \ldots, n\right\}$ by $\operatorname{supp}(u)$.

For any $u \in X^{*}$, we introduce the two following notions of degree:

- the partial degree $|u|_{x}$ of $u$ in $x \in X$ is just the number of $x$ in $u$.

- the multidegree is the $X$-uple $|u|_{X}=\left(|u|_{x}\right)_{x \in X} \in N^{(X)}$, where $N$ is the set of non-negative integers.

Lemma 3.1 Let $u \in X^{*}$ be an $A L S W$ and $x \in X$ such that $x>\operatorname{supp}(u)$. Then, in $\operatorname{Lie}(X),[x u]=[x[u]]=\sum_{i=1}^{m} \alpha_{i}\left(\left(x y_{i}\right) u_{i}\right)$, where for any $i=1, \ldots, m, \alpha_{i} \in k, y_{i} \in X, u_{i} \in$ $X^{*}, \operatorname{supp}(x u)=\operatorname{supp}\left(x y_{i} u_{i}\right),|x u|_{X}=\left|x y_{i} u_{i}\right|_{X}$ and $\overline{\left(\left(x y_{i}\right) u_{i}\right)}=x y_{i} u_{i}$.

Proof We prove the lemma by induction on $|u|$. If $|u|=1$, the result is clear. Let us suppose it has been proved for $|u|<n$ with $n \geq 2$. Let $|u|=n$, and $u=u_{1} u_{2}$ where $u_{2}$ is the longest $A L S W$ proper end of $u$. Then by Lemma 2.2, $u_{1}$ is an $A L S W$ and $[x u]=$ $\left(x\left(\left[u_{1}\right]\left[u_{2}\right]\right)\right)=\left(\left(x\left[u_{1}\right]\right)\left[u_{2}\right]\right)-\left(\left(x\left[u_{2}\right]\right)\left[u_{1}\right]\right)$. By induction, $\left(x\left[u_{1}\right]\right)=\sum_{i=1}^{l} \beta_{i}\left(\left(x y_{i}\right) v_{i}\right)$, where for any $i=1, \ldots, l, \beta_{i} \in k, y_{i} \in X, v_{i} \in X^{*}, \operatorname{supp}\left(x u_{1}\right)=\operatorname{supp}\left(x y_{i} v_{i}\right),\left|x u_{1}\right|_{X}=$ $\left|x y_{i} v_{i}\right|_{X}, \overline{\left(\left(x y_{i}\right) v_{i}\right)}=x y_{i} v_{i}$ and $\left(x\left[u_{2}\right]\right)=\sum_{j=1}^{t} \gamma_{j}\left(\left(x z_{j}\right) w_{j}\right)$, where for any $j=1, \ldots, t, \gamma_{j} \in$ $k, z_{j} \in X, w_{j} \in X^{*}, \operatorname{supp}\left(x u_{2}\right)=\operatorname{supp}\left(x z_{j} w_{j}\right),\left|x u_{2}\right|_{X}=\left|x z_{j} w_{j}\right|_{X}, \overline{\left(\left(x z_{j}\right) w_{j}\right)}=x z_{j} w_{j}$. Then $[x u]=\left(\left(x\left[u_{1}\right]\right)\left[u_{2}\right]\right)-\left(\left(x\left[u_{2}\right]\right)\left[u_{1}\right]\right)=\sum_{i=1}^{l} \beta_{i}\left(\left(\left(x y_{i}\right) v_{i}\right)\left[u_{2}\right]\right)-\sum_{j=1}^{t} \gamma_{j}\left(\left(\left(x z_{j}\right) w_{j}\right)\left[u_{1}\right]\right)$ and $\operatorname{supp}\left(x y_{i} v_{i} u_{2}\right)=\operatorname{supp}\left(x u_{1} u_{2}\right)=\operatorname{supp}(x u), \operatorname{supp}\left(x z_{j} w_{j} u_{1}\right)=\operatorname{supp}\left(x u_{2} u_{1}\right)=\operatorname{supp}(x u)$, $\left|x y_{i} v_{i} u_{2}\right|_{X}=\left|x u_{1} u_{2}\right|_{X}=|x u|_{X},\left|x z_{j} w_{j} u_{1}\right|_{X}=\left|x u_{2} u_{1}\right|_{X}=|x u|_{X}$. Since $x>\operatorname{supp}(u)$ and $u_{1}, u_{2}$ are $A L S W$, we get that $\overline{\left(\left(\left(x y_{i}\right) v_{i}\right)\left[u_{2}\right]\right)}=\overline{\left(\left(x y_{i}\right) v_{i}\right)} \cdot \overline{\left[u_{2}\right]}=x y_{i} v_{i} u_{2}$ and $\overline{\left(\left(\left(x z_{j}\right) w_{j}\right)\left[u_{1}\right]\right)}=$ $\overline{\left(\left(x z_{j}\right) w_{j}\right)} \cdot \overline{\left[u_{1}\right]}=x z_{j} w_{j} u_{1}$.

Lemma 3.2 Let $x, y, z \in X$ and $u, v \in X^{*}$ such that $x>y>\operatorname{supp}(u)$ and $y>z>$ $\operatorname{supp}(v)$. Then the following statements hold.

(i) If $v$ is an $A L S W$, then $(([x u y][v]) z)-([x u]((y[v]) z))=(([x u][v])(y z))+((([x u] z) y)[v])-$ $(([x u](z[v])) y)$.

(ii) Suppose that $v=v_{1} v_{2} \cdots v_{n}$, where $n \geq 2$, $v_{i}$ is an $A L S W, 1 \leq i \leq n$ and $v_{1} \leq v_{2} \leq \cdots \leq v_{n}$. Then

$$
\begin{aligned}
& \left(\left(\left(\left(([x u] y)\left[v_{1}\right]\right)\left[v_{2}\right]\right) \cdots\left[v_{n}\right]\right) z\right)-\left([x u]\left(\left(\left(\left(y\left[v_{1}\right]\right)\left[v_{2}\right]\right) \cdots\left[v_{n}\right]\right) z\right)\right) \\
= & \left(\left(\left(\left(\left(([x u] y)\left[v_{1}\right]\right)\left[v_{2}\right]\right) \cdots\left[v_{n-1}\right]\right) z\right)\left[v_{n}\right]\right)-\left(\left([x u]\left(\left(\left(\left(y\left[v_{1}\right]\right)\left[v_{2}\right]\right) \cdots\left[v_{n-1}\right]\right) z\right)\right)\left[v_{n}\right]\right) \\
& +\left(\left([x u]\left[v_{n}\right]\right)\left(\left(\left(\left(y\left[v_{1}\right]\right)\left[v_{2}\right]\right) \cdots\left[v_{n-1}\right]\right) z\right)\right)-\left(\left([x u]\left(z\left[v_{n}\right]\right)\right)\left(\left(\left(y\left[v_{1}\right]\right)\left[v_{2}\right]\right) \cdots\left[v_{n-1}\right]\right)\right) \\
& -\sum_{i=1}^{n-1}\left(\left(\left(\left(\left([x u]\left[v_{i}\right]\right)\left(\left(\left(\left(y\left[v_{1}\right]\right)\left[v_{2}\right]\right) \cdots\left[v_{i-1}\right]\right)\right)\right)\left[v_{i+1}\right]\right) \cdots\left[v_{n-1}\right]\right)\left(z\left[v_{n}\right]\right)\right) .
\end{aligned}
$$




\section{Proof (i)}

$$
\begin{aligned}
& (([x u y][v]) z)-([x u]((y[v]) z)) \\
= & ((([x u] y)[v]) z)-(([x u](y[v])) z)+(([x u] z)(y[v])) \\
= & ((([x u] y)[v]) z)-((([x u] y)[v]) z)+((([x u][v]) y) z)+((([x u] z) y)[v])-((([x u] z)[v]) y) \\
= & ((([x u][v]) z) y)+(([x u][v])(y z))+((([x u] z) y)[v])-((([x u][v]) z) y)-(([x u](z[v])) y) \\
= & (([x u][v])(y z))+((([x u] z) y)[v])-(([x u](z[v])) y) .
\end{aligned}
$$

(ii) Since

$$
\begin{aligned}
& \left(\left(\left(\left(([x u] y)\left[v_{1}\right]\right)\left[v_{2}\right]\right) \cdots\left[v_{n}\right]\right) z\right) \\
= & \left.\left(\left(\left(([x u] y)\left[v_{1}\right]\right) \cdots\left[v_{n-1}\right]\right) z\right)\left[v_{n}\right]\right)-\left(\left(\left(([x u] y)\left[v_{1}\right]\right) \cdots\left[v_{n-1}\right]\right)\left(z\left[v_{n}\right]\right)\right)
\end{aligned}
$$

and

$$
\begin{aligned}
& \left([x u]\left(\left(\left(y\left[v_{1}\right]\right) \cdots\left[v_{n}\right]\right) z\right)\right) \\
= & \left([x u]\left(\left(\left(y\left[v_{1}\right]\right) \cdots\left[v_{n-1}\right]\right) z\right)\left[v_{n}\right]\right)-\left([x u]\left(\left(y\left[v_{1}\right]\right) \cdots\left[v_{n-1}\right]\right)\left(z\left[v_{n}\right]\right)\right) \\
= & \left(\left([x u]\left(\left(\left(y\left[v_{1}\right]\right) \cdots\left[v_{n-1}\right]\right) z\right)\right)\left[v_{n}\right]\right)-\left(\left([x u]\left[v_{n}\right]\right)\left(\left(\left(y\left[v_{1}\right]\right) \cdots\left[v_{n-1}\right]\right) z\right)\right) \\
& -\left(\left([x u]\left(\left(y\left[v_{1}\right]\right) \cdots\left[v_{n-1}\right]\right)\right)\left(z\left[v_{n}\right]\right)\right)+\left(\left([x u]\left(z\left[v_{n}\right]\right)\right)\left(\left(y\left[v_{1}\right]\right) \cdots\left[v_{n-1}\right]\right)\right) \\
= & \left(\left([x u]\left(\left(\left(y\left[v_{1}\right]\right) \cdots\left[v_{n-1}\right]\right) z\right)\right)\left[v_{n}\right]\right)-\left(\left([x u]\left[v_{n}\right]\right)\left(\left(\left(y\left[v_{1}\right]\right) \cdots\left[v_{n-1}\right]\right) z\right)\right. \\
& -\left(\left(\left([x u]\left(\left(y\left[v_{1}\right]\right) \cdots\left[v_{n-2}\right]\right)\right)\left[v_{n-1}\right]\right)\left(z\left[v_{n}\right]\right)\right)+\left(\left(\left([x u]\left[v_{n-1}\right]\right)\left(\left(y\left[v_{1}\right]\right) \cdots\left[v_{n-2}\right]\right)\right)\left(z\left[v_{n}\right]\right)\right) \\
& +\left(\left([x u]\left(z\left[v_{n}\right]\right)\right)\left(\left(y\left[v_{1}\right]\right) \cdots\left[v_{n-1}\right]\right)\right) \\
= & \left(\left([x u]\left(\left(\left(y\left[v_{1}\right]\right) \cdots\left[v_{n-1}\right]\right) z\right)\right)\left[v_{n}\right]\right)-\left(\left([x u]\left[v_{n}\right]\right)\left(\left(\left(y\left[v_{1}\right]\right) \cdots\left[v_{n-1}\right]\right) z\right)\right. \\
& -\left(\left(\left(\left([x u]\left(\left(y\left[v_{1}\right]\right) \cdots\left[v_{n-3}\right]\right)\right)\left[v_{n-2}\right]\right)\left[v_{n-1}\right]\right)\left(z\left[v_{n}\right]\right)\right) \\
& +\left(\left(\left(\left([x u]\left[v_{n-2}\right]\right)\left(\left(y\left[v_{1}\right]\right) \cdots\left[v_{n-3}\right]\right)\right)\left[v_{n-1}\right]\right)\left(z\left[v_{n}\right]\right)\right) \\
& +\left(\left(\left([x u]\left[v_{n-1}\right]\right)\left(\left(y\left[v_{1}\right]\right) \cdots\left[v_{n-2}\right]\right)\right)\left(z\left[v_{n}\right]\right)\right)+\left(\left([x u]\left(z\left[v_{n}\right]\right)\right)\left(\left(y\left[v_{1}\right]\right) \cdots\left[v_{n-1}\right]\right)\right) \\
= & \cdots \cdots \\
= & \left(\left([x u]\left(\left(\left(y\left[v_{1}\right]\right) \cdots\left[v_{n-1}\right]\right) z\right)\right)\left[v_{n}\right]\right)-\left(\left([x u]\left[v_{n}\right]\right)\left(\left(\left(y\left[v_{1}\right]\right) \cdots\left[v_{n-1}\right]\right) z\right)\right. \\
& -\left(\left(\left(([x u] y)\left[v_{1}\right]\right) \cdots\left[v_{n-1}\right]\right)\left(z\left[v_{n}\right]\right)\right)+\left(\left([x u]\left(z\left[v_{n}\right]\right)\right)\left(\left(y\left[v_{1}\right]\right) \cdots\left[v_{n-1}\right]\right)\right) \\
& +\sum_{i=1}^{n-1}\left(\left(\left(\left(\left([x u]\left[v_{i}\right]\right)\left(\left(\left(\left(y\left[v_{1}\right]\right)\left[v_{2}\right]\right) \cdots\left[v_{i-1}\right]\right)\right)\right)\left[v_{i+1}\right]\right) \cdots\left[v_{n-1}\right]\right)\left(z\left[v_{n}\right]\right)\right),
\end{aligned}
$$

we can get the result.

Theorem 3.3 Let Lie $(X)$ be the free Lie algebra generated by $X$ over a commutative ring $K$ with unit. Then with deg-lex ordering on $X^{*}$, the set $S=\{[x u y] \mid x, y, z \in$ $\left.X, u \in X^{*}, x \triangleright y \triangleright \operatorname{supp}(u)\right\}$ forms a Gröbner-Shirshov basis in Lie $(X)$. As a result, $\operatorname{Irr}(S)=\left\{[u] \mid[u]\right.$ is a $\left.N L S W, u \neq a \bar{s} b, s \in S, a, b \in X^{*}\right\}$ is a K-basis of the free partially commutative Lie algebra Lie $(X \mid \vartheta)=\operatorname{Lie}(X \mid S)$.

Proof Let us check all the possible compositions. The ambiguities $w$ of all possible compositions are:

(i) $w=x u z v y, x, y, z \in X, u, v \in X^{*}, x \triangleright y \triangleright \operatorname{supp}(u z v), x \triangleright z \triangleright \operatorname{supp}(u)$. 
(ii) $w=\left.x u\right|_{x_{1} u_{1} y_{1}} y, x, y, x_{1}, y_{1} \in X, u, u_{1} \in X^{*}, x \triangleright y \triangleright \operatorname{supp}(u), x_{1} \triangleright y_{1} \triangleright \operatorname{supp}\left(u_{1}\right)$.

(iii) $w=x u y v z, x, y, z \in X, u, v \in X^{*}, x \triangleright y \triangleright \operatorname{supp}(u), y \triangleright z \triangleright \operatorname{supp}(v)$.

Now we prove that all the compositions are trivial.

For (i), let $f=[x u z v y], g=[x u z], x, y, z \in X, u, v \in X^{*}, x \triangleright y \triangleright \operatorname{supp}(u z v), x \triangleright$ $z \triangleright \operatorname{supp}(u)$. Suppose $v=v_{1} v_{2} \cdots v_{n}$, where $v_{i}$ is an $A L S W, 1 \leq i \leq n$, and $v_{1} \leq$ $v_{2} \leq \cdots \leq v_{n}$. Then $w=x u z v y$ and $(f, g)_{w}=[x u z v y]-[x u z v y]_{x u z}=([x u z v] y)-$ $\left(\left(\left(\left([x u z]\left[v_{1}\right]\right)\left[v_{2}\right]\right) \cdots\left[v_{n}\right]\right) y\right)=\left([x u z v]-\left(\left(\left([x u z]\left[v_{1}\right]\right)\left[v_{2}\right]\right) \cdots\left[v_{n}\right]\right) y\right)$. By Lemmas 2.4 and 2.3, we have that $[x u z v]-\left(\left(\left([x u z]\left[v_{1}\right]\right)\left[v_{2}\right]\right) \cdots\left[v_{n}\right]\right)=\sum_{i=1}^{m} \alpha_{i}\left[x w_{i}\right]$ where $\alpha_{i} \in k, x w_{i}$ is an $A L S W, \operatorname{supp}\left(x w_{i}\right)=\operatorname{supp}(x u z v),\left|x w_{i}\right|_{X}=|x u z v|_{X}$ and $\overline{\left[x w_{i}\right]}<x u z v$ for any $i=1,2, \ldots m$. Then

$$
(f, g)_{w}=\left(\left(\sum_{i=1}^{m} \alpha_{i}\left[x w_{i}\right]\right) y\right)=\sum_{i=1}^{m} \alpha_{i}\left(\left[x w_{i}\right] y\right)=\sum_{i=1}^{m} \alpha_{i}\left[x w_{i} y\right] \equiv 0 \bmod (S, w) .
$$

For (ii), let $f=\left[\left.x u\right|_{x_{1} u_{1} y_{1}} y\right], g=\left[x_{1} u_{1} y_{1}\right], x, y, x_{1}, y_{1} \in X, u, u_{1} \in X^{*}, x \triangleright y \triangleright$ $\operatorname{supp}(u), x_{1} \triangleright y_{1} \triangleright \operatorname{supp}\left(u_{1}\right)$. Then $w=\left.x u\right|_{x_{1} u_{1} y_{1}} y$ and $(f, g)_{w}=\left[\left.x u\right|_{x_{1} u_{1} y_{1}} y\right]-\left[\left.x u\right|_{x_{1} u_{1} y_{1}} y\right]_{x_{1} u_{1} y_{1}}=$ $\left(\left[\left.x u\right|_{x_{1} u_{1} y_{1}}\right] y\right)-\left(\left(\left[\left.x u\right|_{x_{1} u_{1} y_{1}}\right]_{x_{1} u_{1} y_{1}}\right) y\right)=\left(\left(\left[\left.x u\right|_{x_{1} u_{1} y_{1}}\right]-\left(\left[\left.x u\right|_{x_{1} u_{1} y_{1}}\right]_{x_{1} u_{1} y_{1}}\right)\right) y\right)$. By Lemmas 2.4 and 2.3, we have that $\left[\left.x u\right|_{x_{1} u_{1} y_{1}}\right]-\left(\left[\left.x u\right|_{x_{1} u_{1} y_{1}}\right]_{x_{1} u_{1} y_{1}}\right)=\sum_{i=1}^{l} \alpha_{i}\left[x w_{i}\right]$ where $\alpha_{i} \in k, x w_{i}$ is an $A L S W, \operatorname{supp}\left(x w_{i}\right)=\operatorname{supp}\left(\left.x u\right|_{x_{1} u_{1} y_{1}}\right),\left|x w_{i}\right|_{X}=\left.|x u|_{x_{1} u_{1} y_{1}}\right|_{X}$ and $\overline{\left[x w_{i}\right]}<\left.x u\right|_{x_{1} u_{1} y_{1}}$ for any $i=1,2, \ldots l$. Then

$$
(f, g)_{w}=\left(\left(\sum_{i=1}^{l} \alpha_{i}\left[x w_{i}\right]\right) y\right)=\sum_{i=1}^{l} \alpha_{i}\left(\left[x w_{i}\right] y\right)=\sum_{i=1}^{l} \alpha_{i}\left[x w_{i} y\right] \equiv 0 \bmod (S, w) .
$$

For (iii), let $f=[x u y], g=[y v z], x, y, z \in X, u, v \in X^{*}, x \triangleright y \triangleright \operatorname{supp}(u), y \triangleright z \triangleright \operatorname{supp}(v)$. There are two cases to consider.

(a) $|v|=0$. Then $w=x u y z$ and $(f, g)_{w}=[x u y z]_{x u y}-[x u y z]_{y z}=(([x u] y) z)-$ $([x u](y z))=(([x u] y) z)-(([x u] y) z)+(([x u] z) y)=(([x u] z) y)$. By Lemmas 2.4 and 2.3. we have that $([x u] z)=\sum_{i=1}^{l} \alpha_{i}\left[x w_{i}\right]$, where $\alpha_{i} \in k, x w_{i}$ is an $A L S W, \operatorname{supp}\left(x w_{i}\right)=$ $\operatorname{supp}(x u z),\left|x w_{i}\right|_{X}=|x u z|_{X}$ and $\overline{\left[x w_{i}\right]} \leq \overline{([x u] z)}=x u z$ for any $i=1,2, \ldots l$. Then

$$
(f, g)_{w}=\left(\left(\sum_{i=1}^{l} \alpha_{i}\left[x w_{i}\right]\right) y\right)=\sum_{i=1}^{l} \alpha_{i}\left(\left[x w_{i}\right] y\right)=\sum_{i=1}^{l} \alpha_{i}\left[x w_{i} y\right] \equiv 0 \bmod (S, w) .
$$

(b) $|v| \geq 1$. Suppose $v=v_{1} v_{2} \cdots v_{n}$, where $v_{i}$ is an $A L S W, \quad 1 \leq i \leq n$, and $v_{1} \leq v_{2} \leq \cdots \leq v_{n}$. Then $w=x u y v z$ and by Lemma 3.2, we have that 


$$
\begin{aligned}
& (f, g)_{w}=[x u y v z]_{x u y}-[x u y v z]_{y v z} \\
& =\left(\left(\left(\left(([x u] y)\left[v_{1}\right]\right)\left[v_{2}\right]\right) \cdots\left[v_{n}\right]\right) z\right)-\left([x u]\left(\left(\left(\left(y\left[v_{1}\right]\right)\left[v_{2}\right]\right) \cdots\left[v_{n}\right]\right) z\right)\right) \\
& =\left(\left(\left(\left(\left(([x u] y)\left[v_{1}\right]\right)\left[v_{2}\right]\right) \cdots\left[v_{n-1}\right]\right) z\right)\left[v_{n}\right]\right)-\left(\left([x u]\left(\left(\left(\left(y\left[v_{1}\right]\right)\left[v_{2}\right]\right) \cdots\left[v_{n-1}\right]\right) z\right)\right)\left[v_{n}\right]\right) \\
& +\left(\left([x u]\left[v_{n}\right]\right)\left(\left(\left(\left(y\left[v_{1}\right]\right)\left[v_{2}\right]\right) \cdots\left[v_{n-1}\right]\right) z\right)\right)-\left(\left([x u]\left(z\left[v_{n}\right]\right)\right)\left(\left(\left(y\left[v_{1}\right]\right)\left[v_{2}\right]\right) \cdots\left[v_{n-1}\right]\right)\right) \\
& -\sum_{i=1}^{n-1}\left(\left(\left(\left(\left([x u]\left[v_{i}\right]\right)\left(\left(\left(\left(y\left[v_{1}\right]\right)\left[v_{2}\right]\right) \cdots\left[v_{i-1}\right]\right)\right)\right)\left[v_{i+1}\right]\right) \cdots\left[v_{n-1}\right]\right)\left(z\left[v_{n}\right]\right)\right) \\
& =\left(\left(\left(\left(\left(([x u] y)\left[v_{1}\right]\right) \cdots\left[v_{n-2}\right]\right) z\right)\left[v_{n-1}\right]\right)\left[v_{n}\right]\right)-\left(\left(\left([x u]\left(\left(\left(y\left[v_{1}\right]\right) \cdots\left[v_{n-2}\right]\right) z\right)\right)\left[v_{n-1}\right]\right)\left[v_{n}\right]\right) \\
& +\left(\left(\left([x u]\left[v_{n-1}\right]\right)\left(\left(\left(y\left[v_{1}\right]\right) \cdots\left[v_{n-2}\right]\right) z\right)\right)\left[v_{n}\right]\right)-\left(\left(\left([x u]\left(z\left[v_{n-1}\right]\right)\right)\left(\left(y\left[v_{1}\right]\right) \cdots\left[v_{n-2}\right]\right)\right)\left[v_{n}\right]\right) \\
& \left.-\sum_{i=1}^{n-2}\left(\left(\left(\left(\left((x u]\left[v_{i}\right]\right)\left(\left(\left(y\left[v_{1}\right]\right) \cdots\left[v_{i-1}\right]\right)\right)\right)\left[v_{i+1}\right]\right) \cdots\left[v_{n-2}\right]\right)\left(z\left[v_{n-1}\right]\right)\right)\left[v_{n}\right]\right) \\
& +\left(\left([x u]\left[v_{n}\right]\right)\left(\left(\left(y\left[v_{1}\right]\right) \cdots\left[v_{n-1}\right]\right) z\right)\right)-\left(\left([x u]\left(z\left[v_{n}\right]\right)\right)\left(\left(y\left[v_{1}\right]\right) \cdots\left[v_{n-1}\right]\right)\right) \\
& -\sum_{i=1}^{n-1}\left(\left(\left(\left(\left([x u]\left[v_{i}\right]\right)\left(\left(\left(y\left[v_{1}\right]\right) \cdots\left[v_{i-1}\right]\right)\right)\right)\left[v_{i+1}\right]\right) \cdots\left[v_{n-1}\right]\right)\left(z\left[v_{n}\right]\right)\right) \\
& =\cdots \cdots \text {. } \\
& =\left(\left(\left(\left([x u y]\left[v_{1}\right]\right) z\right)\left[v_{2}\right]\right) \cdots\left[v_{n}\right]\right)-\left(\left(\left([x u]\left(\left(y\left[v_{1}\right]\right) z\right)\right)\left[v_{2}\right]\right) \cdots\left[v_{n}\right]\right) \\
& +\left(\left(\left(\left([x u]\left[v_{2}\right]\right)\left(\left(y\left[v_{1}\right]\right) z\right)\right)\left[v_{3}\right]\right) \cdots\left[v_{n}\right]\right)-\left(\left(\left(\left([x u]\left(z\left[v_{2}\right]\right)\right)\left(y\left[v_{1}\right]\right)\right)\left[v_{3}\right]\right) \cdots\left[v_{n}\right]\right) \\
& \left.-\left(\left(\left(\left([x u]\left[v_{1}\right]\right) y\right)\left(z\left[v_{2}\right]\right)\right)\left[v_{3}\right]\right) \cdots\left[v_{n}\right]\right)+\cdots \cdots \\
& +\left(\left(\left([x u]\left[v_{n-1}\right]\right)\left(\left(\left(y\left[v_{1}\right]\right) \cdots\left[v_{n-2}\right]\right) z\right)\right)\left[v_{n}\right]\right)-\left(\left(\left([x u]\left(z\left[v_{n-1}\right]\right)\right)\left(\left(y\left[v_{1}\right]\right) \cdots\left[v_{n-2}\right]\right)\right)\left[v_{n}\right]\right) \\
& -\sum_{i=1}^{n-2}\left(\left(\left(\left(\left(\left([x u]\left[v_{i}\right]\right)\left(\left(\left(y\left[v_{1}\right]\right) \cdots\left[v_{i-1}\right]\right)\right)\right)\left[v_{i+1}\right]\right) \cdots\left[v_{n-2}\right]\right)\left(z\left[v_{n-1}\right]\right)\right)\left[v_{n}\right]\right) \\
& +\left(\left([x u]\left[v_{n}\right]\right)\left(\left(\left(y\left[v_{1}\right]\right) \cdots\left[v_{n-1}\right]\right) z\right)\right)-\left(\left([x u]\left(z\left[v_{n}\right]\right)\right)\left(\left(y\left[v_{1}\right]\right) \cdots\left[v_{n-1}\right]\right)\right) \\
& -\sum_{i=1}^{n-1}\left(\left(\left(\left(\left([x u]\left[v_{i}\right]\right)\left(\left(\left(y\left[v_{1}\right]\right) \cdots\left[v_{i-1}\right]\right)\right)\right)\left[v_{i+1}\right]\right) \cdots\left[v_{n-1}\right]\right)\left(z\left[v_{n}\right]\right)\right) \\
& =\left(\left(\left(\left([x u]\left[v_{1}\right]\right)(y z)\right)\left[v_{2}\right]\right) \cdots\left[v_{n}\right]\right)+\left(\left(\left((([x u] z) y)\left[v_{1}\right]\right)\left[v_{2}\right]\right) \cdots\left[v_{n}\right]\right) \\
& -\left(\left(\left(\left([x u]\left(z\left[v_{1}\right]\right)\right) y\right)\left[v_{2}\right]\right) \cdots\left[v_{n}\right]\right)+\left(\left(\left(\left([x u]\left[v_{2}\right]\right)\left(\left(y\left[v_{1}\right]\right) z\right)\right)\left[v_{3}\right]\right) \cdots\left[v_{n}\right]\right) \\
& -\left(\left(\left(\left([x u]\left(z\left[v_{2}\right]\right)\right)\left(y\left[v_{1}\right]\right)\right)\left[v_{3}\right]\right) \cdots\left[v_{n}\right]\right)-\left(\left(\left(\left(\left([x u]\left[v_{1}\right]\right) y\right)\left(z\left[v_{2}\right]\right)\right)\left[v_{3}\right]\right) \cdots\left[v_{n}\right]\right) \\
& +\cdots \cdots+ \\
& +\left(\left(\left([x u]\left[v_{n-1}\right]\right)\left(\left(\left(y\left[v_{1}\right]\right) \cdots\left[v_{n-2}\right]\right) z\right)\right)\left[v_{n}\right]\right) \\
& -\left(\left(\left([x u]\left(z\left[v_{n-1}\right]\right)\right)\left(\left(y\left[v_{1}\right]\right) \cdots\left[v_{n-2}\right]\right)\right)\left[v_{n}\right]\right) \\
& -\sum_{i=1}^{n-2}\left(\left(\left(\left(\left(\left([x u]\left[v_{i}\right]\right)\left(\left(\left(y\left[v_{1}\right]\right) \cdots\left[v_{i-1}\right]\right)\right)\right)\left[v_{i+1}\right]\right) \cdots\left[v_{n-2}\right]\right)\left(z\left[v_{n-1}\right]\right)\right)\left[v_{n}\right]\right) \\
& +\left(\left([x u]\left[v_{n}\right]\right)\left(\left(\left(y\left[v_{1}\right]\right) \cdots\left[v_{n-1}\right]\right) z\right)\right)-\left(\left([x u]\left(z\left[v_{n}\right]\right)\right)\left(\left(y\left[v_{1}\right]\right) \cdots\left[v_{n-1}\right]\right)\right) \\
& \left.-\sum_{i=1}^{n-1}\left(\left(\left(\left((x u]\left[v_{i}\right]\right)\left(\left(\left(y\left[v_{1}\right]\right) \cdots\left[v_{i-1}\right]\right)\right)\right)\left[v_{i+1}\right]\right) \cdots\left[v_{n-1}\right]\right)\left(z\left[v_{n}\right]\right)\right) \\
& \equiv 0 \bmod (S, w) \text {. }
\end{aligned}
$$

So, all compositions in $S$ are trivial. 
Clearly, Lie $(X \mid \vartheta)=\operatorname{Lie}(X \mid S)$.

The latter conclusion follows from the Composition-Diamond lemma for Lie algebras (Lemma 2.6).

Acknowledgement: The authors would like to thank Professor L.A. Bokut for his guidance, useful discussions and enthusiastic encouragement in writing up this paper.

\section{References}

[1] L.A. Bokut and Yuqun Chen, Gröbner-Shirshov basis for free Lie algebras: after A.I. Shirshov, Southeast Asian Bull. Math., 31, 1057-1076 (2007).

[2] L.A. Bokut and L.S. Shiao, Gröbner-Shirshov bases for Coxeter groups, Comm. in Algebra, 29(9), 4305-4319 (2001).

[3] P. Cartier and D. foara, Problèmes combinatoires de commutation et de rèarrangements, Lecture Notes in Mathematics, $\mathbf{8 5}$, Springer-Verlag, Berlin/New York, (1969).

[4] K.T. Chen, R.H. Fox and R.C. Lyndon, Free differential calculus, IV: the quotient groups of the lower central series, Annals of Mathematics, 68, 81-95 (1958).

[5] C. Choffrut, Free partially commutative monoids, LITP Report, Paris, 86-20 (1986).

[6] V. Diekert, Combinatorics on traces, Lect. Notes in Cpmut. Sci., 454, Springer, (1990).

[7] C. Duboc, Commutations dans les monoìdes libres: Un cadre théorique pour l'étude du parallálisme, Thèse d'Université, Université de Rouen, LITP Report No, 86-25 (1986).

[8] G. Duchamp, Algorithms sur les polynômes en variables non commutatives, Thèse d'Université, Université Paris 7, LITP Report No, 87-58 (1987).

[9] G. Duchamp, The Free Partially Commutative Lie Algebra: Bases and Ranks, Advances in Mathematics, 95, 92-126 (1992).

[10] G. Duchamp and D. Krob, Free Partially Commutative Structures, Journal of Algebra, 156, 318-361 (1993).

[11] E.S. Esyp, I.V. Kazatchkov and V.N. Remeslennikov, Divisibility theory and complexity of algorithms for free partially commutative groups, Contemp. Math., Amer. Math. Soc., 317-346 (2004).

[12] R.C. Lyndon, On Burnside's problem, Trans. Am. math. Soc., 77, 202-215 (1954).

[13] D. Perrin, Partial commutations, [in "Proceedings of 16th ICALP"], Lect. Notes in Comput. Sci., 372, 637-651, Springer, (1989).

[14] E.N. Poroshenko, Bases for partially commutative Lie algebras, arxiv.org/abs/1012.1089v1. 
[15] W. Schmitt, Hopf algebras and identities in free partially commutative monoids, Theoret. Compui. Sci., 73, 335-340 (1990).

[16] A.I. Shirshov, Some algorithmic problem for Lie algebras, Sibirsk. Mat. Z., 3, 292-296 (1962) (in Russian); English translation in SIGSAM Bull., 33(2), 3-6 (1999).

[17] Selected works of A.I. Shirshov, Eds L.A. Bokut, V. Latyshev, I. Shestakov, E. Zelmanov, Trs M. Bremner, M. Kochetov, Birkhäuser, Basel, Boston, Berlin, 2009.

[18] J.Y. Thibon, Intégrité des algèbres de séries formelles sur un alphabet partiellement commutatif, Theorer. Comput. Sci., 41, (1985).

[19] V.A. Ufnarovski, Combinatorial and Asymptotic Methods in Algebra, Encyclopaedia Mat. Sci., 57, 1-196 (1995). 